\title{
Management of Elderly Patients With Gliomas
}

\author{
Jaime Gállego Pérez-LARraya, a JeAn-YVes Delattre ${ }^{b, c, d}$ \\ ${ }^{a}$ Department of Neurology, Clínica Universidad de Navarra, Universidad de Navarra, Pamplona, Spain; ${ }^{b}$ Service de Neurologie 2, Division

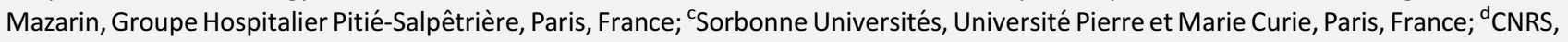 \\ UMR 7225, INSERM, Paris, France \\ Disclosures of potential conflicts of interested may be found at the end of this article.
}

Key Words. Glioma • Low-grade glioma • High-grade glioma • Glioblastoma • Elderly • Old patients

\section{ABSTRACT}

The current progressive aging of the population is resulting in a continuous increase in the incidence of gliomas in elderly people, especially the most frequent subtype, glioblastoma (GBM). This sociohealth shift, known as the "silver tsunami," has prompted the neuro-oncology community to investigate the role of specific antitumor treatments, such as surgery, radiotherapy, chemotherapy, and other targeted therapies, for these traditionally undertreated patients. Advanced age, a widely recognized poor prognostic factor in both low-grade glioma (LGG) and high-grade glioma patients, should no longer be the sole reason for excluding such older patients from receiving etiologic treatments. Far from it, results from recent prospective trials conducted on elderly patients with GBM demonstrate that active management of these patients can have a positive impact on survival without impairing either cognition or quality of life. Although prospective studies specifically addressing the management of grade 2 and 3 gliomas are lacking and thus needed, the aforementioned tendency toward acknowledging a therapeutic benefit for GBM patients might also apply to the treatment of patients with LGG and anaplastic gliomas. In order to optimize such etiologic treatment in conjunction with symptomatic management, neuro-oncology multidisciplinary boards must individually consider important features such as resectability of the tumor, functional and cognitive status, associated comorbidities, and social support. The Oncologist 2014;19:1258-1267

Implications for Practice: The progressive aging of the population is resulting in an increase in the incidence of gliomas, more particularly glioblastoma, among elderly people. Therefore the role of specific therapeutic regimens for these traditionally undertreated patients has become a challenging and contemporary topic for neuro-oncologists. Recent studies demonstrate that active management can have a positive impact on survival without impairing either cognition or quality of life. Importantly, in order to optimize such etiologic treatment in conjunction with symptomatic management, neuro-oncology multidisciplinary boards should individually consider important features such as functional and cognitive status, associated comorbidities and social support.

\section{INTRODUCTION}

Life expectancy has consistently increased over the past few decades. As a consequence, the proportion of the elderly population has exploded and is expected to keep rising in the coming years, leading to the sociological phenomenon called the "silver tsunami." Because older people are more prone to disease, there is an emerging need for medical innovation to address the health problems that arise from this situation [1-3].

Particularly, age is one of the strongest risk factors for cancer. Moreover, cancer is especially complex for older adults because a number of factors need to be taken into account when understanding their prognoses and formulating their care plans to handle other issues, such as altered physiology, neurocognitive impairment, multiple comorbidities, increased treatment-related adverse effects, different goals of care, and the increased importance of social support [3].

This sociohealth context is applicable to gliomas, the most frequent malignant primary brain tumor in adults [4]. Over the past few decades the incidence of these brain tumors, in particular the most frequent subtype, glioblastoma (GBM), has increased in the older segments of the population [5-8], although the trend has recently stabilized.

Nowadays, GBM is primarily diagnosed at older ages, with a median age at diagnosis of 64 years. Hence, older patients already account for nearly half of all patients suffering from GBM [4]. In this sense, the continuous aging of the population will lead to a significant increase (at least twofold) in the number of cases of GBM in elderly patients in the following two decades [9-11].

Until recently, as happened with other cancers in which age is a well-recognized pejorative prognostic factor [12], elderly patients with gliomas, particularly GBM, were excluded from clinical trials [13-15] and from effective therapies because of the preconceived ideas that such therapies would lack efficacy and that patients would be unable to tolerate the treatment.

Correspondence: Jean-Yves Delattre, M.D., Service de Neurologie 2, Division Mazarin, Groupe Hospitalier Pitié-Salpêtrière, 47-83 Boulevard de I'Hôpital, 75013 Paris, France. Telephone: 33-01-42-160-385; E-Mail: jean-yves.delattre@psl.aphp.fr Received August 28, 2014; accepted for publication September 11, 2014; first published online in The Oncologist Express on October 23, 2014. CAlphaMed Press 1083-7159/2014/ $\$ 20.00 / 0$ http://dx.doi.org/10.1634/theoncologist.2014-0170 
As a consequence, these patients were far less likely to undergo resection and receive adjuvant therapies than their younger counterparts and were typically offered only palliative care [16-19]. Fortunately, as a result of the aforementioned demographic shifts, management of these tumors in elderly patients has become an important and challenging topic in neuro-oncology. The scientific community has apprehended that an advanced age cannot be the sole reason for depriving patients of an active therapeutic approach [20]. Because it is no longer acceptable to continue undertreating or improperly managing nearly half of the patients with GBM without relying on data from evidence-based medicine, important efforts have been made in the last decade to conduct clinical trials aimed at analyzing the impact of different therapeutic alternatives, not only in survival and toxicity but also in quality of life and cognition [21].

The purpose of this article is to provide a comprehensive review of the current symptomatic and etiologic management of both low-grade gliomas (LGGs) and high-grade gliomas (HGGs) in elderly patients, with special emphasis on recent clinical trials that have led to a shift from a nihilist therapeutic attitude toward an active evidence-based practice for this population. The boundary between young and old age cannot be exactly defined, because chronological age does not necessarily match physiological age and because it shifts according to society and each individual's health context. Although the definition of elderly varies from study to study and the classification into different subgroups such as "young-old" and "old-old" adults has been advocated, the cutoff of 65-70 years is generally accepted for practical purposes and is used in this review.

\section{Symptomatic Management}

Neurocognitive impairment, affective or mood disorders, seizures, thromboembolic complications, and fatigue frequently occur among elderly patients with gliomas. The aim of supportive care is to relieve these symptoms of the disease, which can lead to increased comorbidity, impaired quality of life, and mortality [22]. In consequence, symptomatic management must be provided to every elderly patient suffering from gliomas [23]. Such treatment consists mainly of corticosteroids and antiepileptic drugs.

Corticosteroids are the pillar of treatment for symptomatic peritumoral edema, which is more commonly present in HGG patients. They relieve neurological focal deficits secondary to edema, as well as symptoms derived from intracranial hypertension such as headache and vomiting. However, corticosteroids are associated with a wide range of harmful side effects, which can be more pronounced in older patients [24, 25]. Osteoporosis, muscular and skin atrophy, adverse psychiatric events, and lymphopenia-related pneumocystosis seem to be particularly frequent in this population. Therefore, steroid treatment should be given only to symptomatic patients at the lowest possible dose and for the shortest duration of time [26]. Additionally, prophylactic measures aimed at reducing the risk of such deleterious effects should be seriously considered in these patients, particularly potassium, calcium and vitamin D supplements, bisphosphonate therapy, gastrointestinal ulcer prophylaxis with a gastric acid inhibitor, and trimethoprim/sulfamethoxazole for prevention of Pneumocystis carinii pneumonia.

Epilepsy is another common complication of both LGGs and HGGs in the elderly, although its presentation might be subtler than in younger patients, and its presence significantly increases the risk of falls and fractures in this fragile population $[27,28]$.The general principles for the management of epilepsy also apply to elderly patients, with some caveats. Anticonvulsants must be prescribed only to those patients who have suffered seizures [29]. To avoid drug interactions and adverse effects, particularly in the case of comorbidity, antiepileptic drug monotherapy with lamotrigine or levetiracetam is preferable [30, 31]. Lower dosages of anticonvulsants and a longer period of up-titrating are advisable $[27,28]$. On the one hand, older adults appear to be more responsive to antiepileptic drug therapy than their younger counterparts. On the other hand, they are also more likely to experience adverse events at lower serum anticonvulsant concentrations, especially cognitive impairment and imbalance. Thus, elderly patients with glioma-related epilepsy should be monitored closely, with particular attention to avoiding anticonvulsant concentrations in the higher range. Levels below the usually effective range may be appropriate for them [32].

Elderly patients with glial tumors often have reduced mobility and are thus at higher risk of developing deep venous thrombosis and thromboembolic events. Adequate prevention with active and passive mobilization, compression stocking, or low-molecular-weight heparin is commonly needed [21].

In addition to these supportive treatments, affective disorders such as depression or anxiety must be promptly recognized, because patients might also benefit from psychosocial support and specific pharmacotherapy, which must also be introduced at low doses and titrated slowly. Psychostimulants such as methylphenidate might also be used in selected patients to relieve life-limiting fatigue [33-36], which is common in elderly patients with gliomas undergoing radiotherapy and chemotherapy. Again, such treatment must also be initiated at a low dosage and escalated gradually. Physical and cognitive rehabilitation may also have a positive influence on a patient's quality of life [21]. The impact of all these supportive measures on the outcome and quality of life of these fragile patients should be specifically evaluated in further prospective trials.

\section{Low-Grade Glioma}

The exact incidence of LGG in elderly patients is still unknown. Recent reports from brain tumor registries or single-institution series suggest that approximately $8 \%$ of all LGGs occur in this cohort $[37,38]$. However, this observation might be somewhat underestimated because older patients are in fact, as demonstrated by retrospective studies, less likely to undergo surgery for histological confirmation of a suspected LGG on neuroimaging [39].

The clinical picture, course, and management of these tumors in aged patients differ from that in younger patients [37, 40, 41]. Elderly patients present more commonly with focal neurological deficits, such as cognitive impairment, language disorders, and sensorimotor disturbances, rather than with epileptic seizures, possibly reflecting a more accelerated and extended growth of the tumor $[37,41]$. These patients 
commonly have larger tumors on neuroimaging, frequently infiltrating both cerebral hemispheres. Importantly, up to $45 \%$ of LGGs in elderly patients exhibit some type of contrast enhancement, thus mimicking a higher-grade lesion, a figure that is twice the frequency seen in younger patients $[37,41]$. In terms of histopathological and molecular features, the limited data existing in the literature suggest that there are no obvious differences in the distribution of glioma subtypes and rates of $1 p / 19 q$ codeletion in older patients compared with their younger counterparts [37]. Additionally, IDH1 mutations have not been specifically addressed in aged LGG patients.

Elderly patients present more commonly with focal neurological deficits, such as cognitive impairment, language disorders, and sensorimotor disturbances, rather than with epileptic seizures, possibly reflecting a more accelerated and extended growth of the tumor.

Because prospective trials have not specifically addressed this issue, controversy exists about the optimal management of LGG in this population, including the role of surgery and its extension, the optimal timing for radiotherapy, and the utility of chemotherapy. As a consequence of this lack of evidence, older patients with suspected LGGs have traditionally had a much lower likelihood of undergoing tumor resection and receiving standard treatment with radiotherapy than younger patients $[39,41]$. More prominent clinical deficits and tumors with larger sizes frequently crossing the midline have been suggested as possible reasons for these figures [37]. The median overall survival (OS) of approximately 3 years and the 5 -year survival rates below $40 \%$ observed in retrospective studies could indicate that LGGs behave more aggressively in elderly patients than in younger ones $[37,40,41]$. Whether this might be explained by a more malignant course of the disease or by the common coexistence of widely recognized pejorative prognostic factors, such as impaired functional status and tumor extension [42], is still a matter of debate. Undertreatment in this population is also an issue. However, despite the lack of specific studies and according to general therapeutic strategies for patients with LGG, these dismal prognostic figures favor the administration of immediate postoperative treatment. Because the neurotoxicity of radiotherapy increases with age, we believe that care should be paid to reduce the irradiation of normal tissues (preferably intensitymodulated radiation therapy whenever possible) as much as possible. Additionally, the dose should be optimized at $45 \mathrm{~Gy}$ administered in 1.8-Gy fractions, because this regimen was found to be as efficient as higher doses in a prospective randomized study in younger patients [43]. An alternative strategy is to use chemotherapy with temozolomide because of its good tolerance profile. Indeed, chemotherapy with temozolomide has been preferentially administered in a few recent retrospective studies. However, it is worth noting that in one study, $55 \%$ of these elderly patients who eventually died were never offered radiotherapy [37], an observation compatible with possible undertreatment of this population.
Some high survival rates might be seen, especially in the cases with more favorable prognostic factors, such as "young-old" (65-75 years old) patients, circumscribed or resectable tumors, oligodendroglial histological subtype, and possibly the $1 p / 19 q$ codeletion/IDH1 mutation (Fig. 1). Pending results of the phase III EORTC 22033-26033 study, which is evaluating primary chemotherapy with temozolomide versus radiotherapy in patients with LGG after stratification for genetic $1 p$ loss, could help because there is no age limit in this trial.

\section{AnAPlastic GLIOMA}

Because of its comparatively lower incidence [4], data on anaplastic glioma in elderly patients are very scarce in the literature. Although some recent large prospective clinical trials have been conducted on elderly patients with malignant gliomas in general, the vast majority of the included patients suffered from GBM, and the percentages of patients with anaplastic tumors were merely anecdotic [44, 45]. Thus, these studies solely address the question of optimal management for GBM, and their results preclude obtaining firm conclusions about the management of grade 3 gliomas in older patients.

According to recent retrospectives studies focusing on this issue, the clinicoradiological and histological pictures of anaplastic gliomas in the elderly do not seem different from those in younger patients. With the limitations of its retrospective nature, a study conducted on 42 older patients with anaplastic astrocytoma ( $A A)$ suggested that tumor resection and sparse instead of diffuse enhancement were associated with longer OS. Adding chemotherapy to radiotherapy was also associated with a prolonged survival but carried important complication risks [46]. As a consequence of the lack of prospective specific studies, the treatment of AA has not yet been standardized for the elderly. Thus, its management might still be extrapolated from the treatment of elderly patients with GBM, taking into account the functional status and comprehensive geriatric assessment (Fig. 2).

Therapeutic decisions might be even more difficult in elderly patients with anaplastic oligodendrogliomas or oligoastrocytomas because of the paucity of data. For example, the results obtained from studies of younger populations support the combination of radiation therapy and chemotherapy with the classic procarbazine, lomustine, and vincristine (PCV) regimen in $1 p / 19 q$ codeleted oligodendroglial tumors [47], but this approach may be inadequate because of the increased risk of neurotoxicity from both radiotherapy and PCV in the elderly. Upfront temozolomide might represent a reasonable alternative in this selected population. A singleinstitution retrospective series suggested that upfront temozolomide induces a response in a substantial number of tumors, with a $20 \%$ rate of grade 3 or 4 hematologic toxicity in elderly patients with anaplastic oligodendroglial tumors. The rate of responders was similar in patients with both $O^{6}$. methylguanine-DNA methyltransferase (MGMT) promoter methylated and unmethylated status, respectively, but the duration of response was significantly longer in the former than in the latter [48].

\section{GLIOBLASTOMA}

GBM is the most frequent malignant primary brain tumor in elderly patients [4], who commonly present with a relatively 

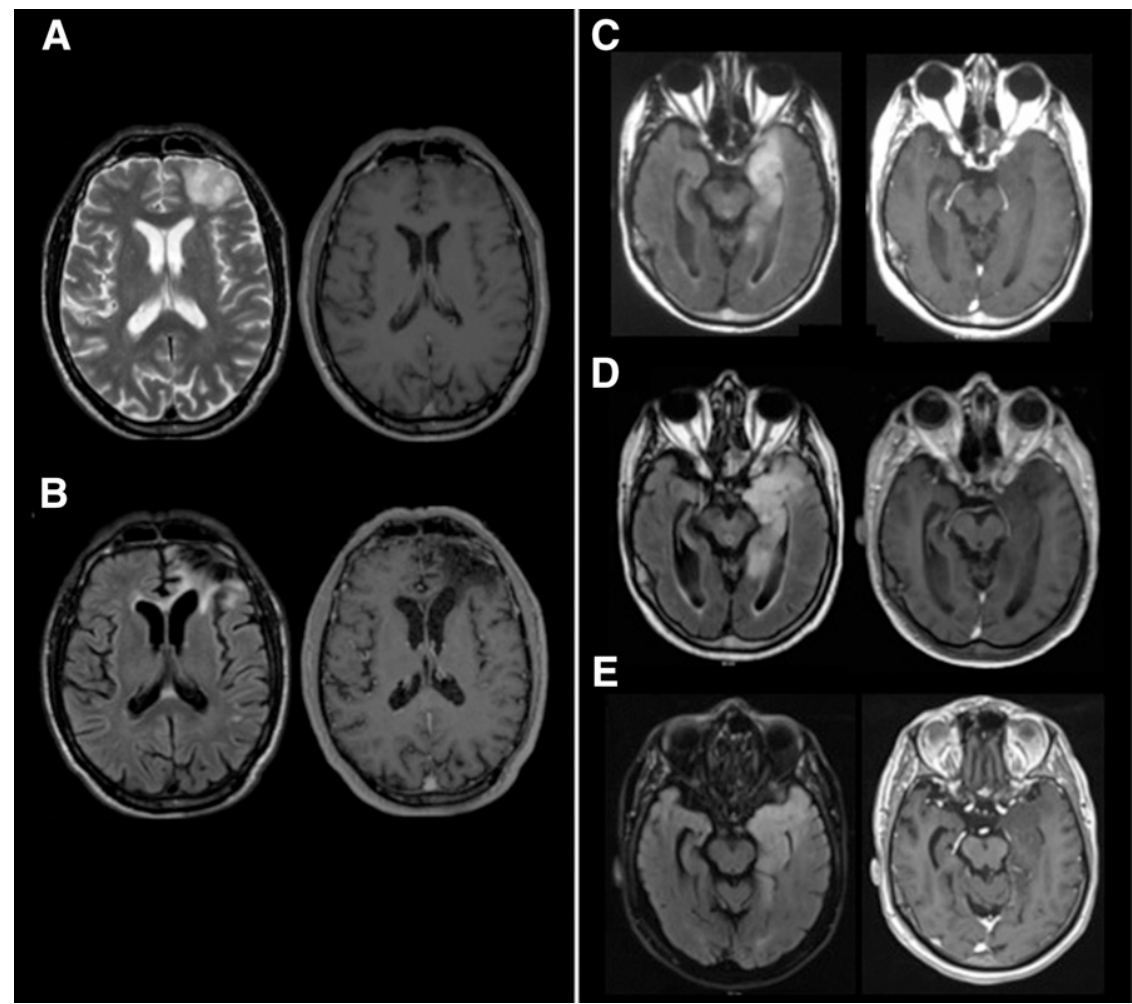

Figure 1. Positive examples of elderly patients with grade 2 gliomas. (A): Presurgical magnetic resonance imaging (MRI) of a 70-year-old man who presented with seizures. The patient underwent surgical resection, and definite diagnosis consisted of $1 p / 19 q$ codeleted, IDH1 mutated grade 2 oligodendroglioma. No complementary treatment was administered. (B): At last follow-up, 5 years after surgery, the patient is doing well (Karnofsky performance score $[\mathrm{KPS}]=90 \%$ ) with an MRI showing an excellent persistent response. (C): Diagnostic MRI showing an infiltrative left temporal lesion in a 73-year-old patient. A biopsy was performed, and the diagnosis of non-1p/19q codeleted, IDH1 mutated grade 2 astrocytoma was retained. The patient was followed closely without any further treatment. (D): Five years later, radiological signs of progression were observed, and treatment with temozolomide was started. (E): At last follow-up, 4 years after chemotherapy, the patient (who is now 82 years old) is clinically (KPS $=80 \%$ ) and radiologically stable.

rapid symptomatic course over a few weeks, with progressive impairment of functional autonomy and cognition [49]. Based on this premise, substantial clinical research has been developed over the past decade in older patients with this disease, focusing not only on survival benefits but also on quality of life and cognitive issues [21]. As a result, the management of elderly GBM patients has fortunately evolved from a purely supportive approach to specific active therapies against the tumor. The results of these trials assessing specific treatments are discussed below and summarized in Table 1.

\section{Surgery}

As it happens in younger patients, surgery is mandatory for further histological examination of tumor samples. This is the only procedure that allows ruling out other nontumoral and potentially curable lesions, such as abscesses. Also, it permits the correct classification and grading of primary gliomas. This latter feature is particularly relevant in the elderly, because up to $44 \%$ of LGG cases in this population may exhibit some contrast enhancement on magnetic resonance imaging (MRI), thus suggesting the radiological diagnosis of a higher-grade tumor $[37,41]$.

In some exceptional circumstances, such as patients in very poor clinical condition or with severe comorbidities that preclude them from undergoing surgery, a working diagnosis of probable HGG is sometimes made, relying on a standard MRI completed by magnetic resonance spectroscopy and perfusion analyses. However, even when the clinical context and multimodality magnetic resonance neuroimaging are suggestive, the risk of committing a diagnostic mistake still exists, and both the patients and their families must be aware of such a possibility [21].

Traditionally, elderly patients have been thought to recover at a slower rate from surgery and to be at a higher risk of developing postoperative neurologic complications $[50,51]$. Contrary to this impression, recent retrospective studies suggest that older patients tolerate aggressive neurosurgery without increased surgery-related morbidity $[52,53]$. In addition to this observation, these studies also suggest that extensive resection of tumors has a positive impact on survival as compared with simple biopsy [17, 52-56], as recognized in younger patients.

Commonly, the decision of whether to conduct a biopsy or a more extensive surgical resection is based on characteristics of the tumor itself and the associated comorbidities of the older patient [57]. A small prospective Finnish trial confirmed the interest in and effectiveness of extensive resection in patients aged 65 years or older with malignant gliomas, most of them GBM [58]. Patients were assigned to undergo either stereotactic biopsy or open craniotomy and resection of the tumor, followed by radiation therapy in both groups. With the limitation of the reduced sample size, this study already 

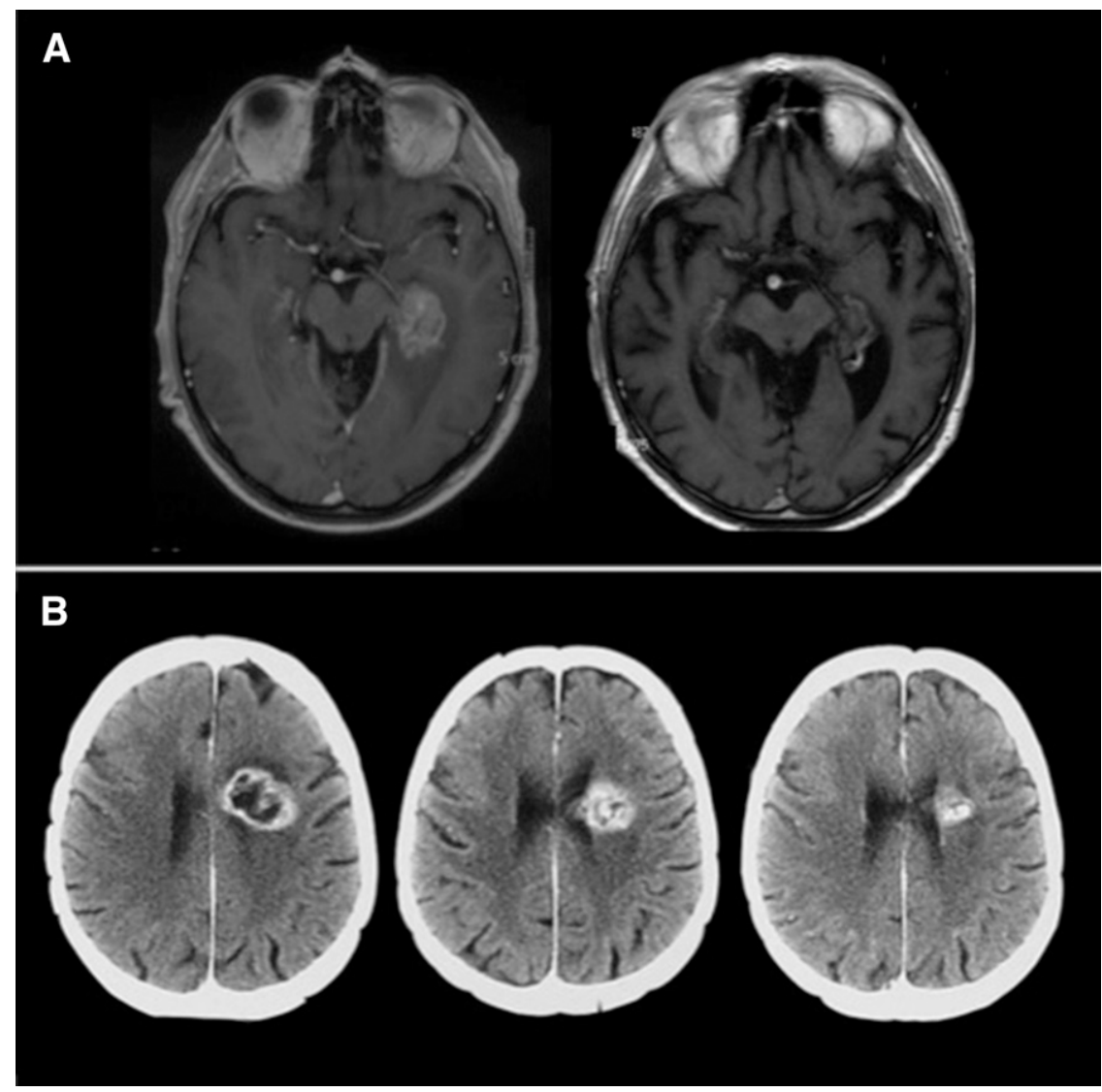

Figure 2. Examples of response in elderly patients with grade 3 gliomas. (A): Left: Presurgical magnetic resonance imaging (MRI) of a 70year-old patient. The patient underwent biopsy of the lesion. Definite diagnosis was non-1p/19q codeleted, IDH1 mutated grade 3 oligodendroglioma. The patient was further treated with radiotherapy with concomitant and adjuvant temozolomide. Right: Brain MRI performed 7 years after diagnosis, demonstrating a persistent complete response. Karnofsky performance score was $80 \%$. (B): Left: Postbiopsy cerebral computed tomography (CT) scan of a 74-year-old patient. Pathological diagnosis was grade 3 oligodendroglioma. Middle: CTscan after 4 cycles of upfront treatment with temozolomide, showing a partial response. Right: CTscan after 12 cycles of upfront treatment with temozolomide, showing a partial response. Progression-free survival was 25 months.

showed that extensive resection was associated with an improved survival (5.6 months) as compared with needle biopsy (2.8 months). Moreover, resection also resulted in a gain in quality of life. Apart from this specific trial, several studies and prospective trials primarily evaluating other therapeutic approaches have also found an independent association between the extent of resection and survival $[17,44,54,59]$.

\section{Radiation Therapy}

A multicenter phase III trial established the role of radiation therapy in the management of elderly patients with GBM [44]. Eighty-one patients aged 70 years or older with newly diagnosed GBM and a Karnofsky performance score (KPS) of $70 \%$ or higher were included in the study. The patients were randomly assigned to receive either supportive care alone or supportive care plus radiotherapy after biopsy or surgical resection. Radiation therapy consisted of fractioned focal irradiation at a dose of $1.8 \mathrm{~Gy}$ per fraction given once daily for 5 days per week for a total dose of $50 \mathrm{~Gy}$. The study was closed at the first interim analysis, showing a clear superiority of the radiotherapy arm. The median survival benefit of adding radiotherapy to the best symptomatic management was 12 weeks (17 weeks for the group of patients receiving only the best supportive care versus 29 weeks for the group of patients who additionally received radiotherapy). Because the goal of treatment was palliation, a particularly important finding of this study was that radiation therapy was well tolerated and did not cause further deterioration in the performance status, health-related quality of life, or cognitive functions.

The aforementioned trial confirmed the benefit of radiotherapy on elderly GBM patients with good performance status, as was previously suggested by a single-institution historical case series $[17,51,60]$. In view of its results, postoperative radiotherapy has become the standard of care for elderly patients with GBM. However, the optimal dose and schedule of radiation therapy remains uncertain. In addition to its associated morbidity, conventional prolonged courses of radiotherapy delivering 60 Gy over a 6-week period might seem too long and inconvenient for some aged patients with short survival expectancy. Hence, short-term radiotherapy has been suggested as an effective and safe alternative for this selected population [60-62]. This issue was specifically addressed in another randomized trial [63]. The study included 100 GBM patients aged 60 years or older. In the postoperative setting, patients were assigned to receive either a conventional course of radiotherapy (60 Gy in 30 fractions over 6 weeks) or a short-course regimen ( $40 \mathrm{~Gy}$ in 15 fractions over 3 weeks). Survival times were similar for the two groups 
Table 1. Selected clinical trials conducted on elderly patients with high-grade gliomas

\begin{tabular}{|c|c|c|c|c|}
\hline Treatment & Trial & Age (years) & KPS (\%) & Conclusions \\
\hline \multicolumn{5}{|l|}{ Surgery } \\
\hline Stereotactic biopsy vs. tumor resection & $\mathrm{R}$ & $\geq 65$ & $\geq 60$ & Increased OS after tumor resection \\
\hline \multicolumn{5}{|l|}{ Radiation therapy } \\
\hline $\begin{array}{l}\text { Standard RT ( } 50 \text { Gy in } 28 \mathrm{fr} \text { ) vs. supportive } \\
\text { care alone }\end{array}$ & $\mathrm{R}$ & $\geq 70$ & $\geq 70$ & $\begin{array}{l}\text { Increased PFS and OS after RT, without } \\
\text { impairing quality of life or cognition }\end{array}$ \\
\hline $\begin{array}{l}\text { Standard (60 Gy in } 30 \mathrm{fr} \text { ) vs. short-course RT } \\
\text { ( } 40 \mathrm{~Gy} \text { in } 15 \mathrm{fr} \text { ) }\end{array}$ & $\mathrm{R}$ & $\geq 60$ & $\geq 50$ & $\begin{array}{l}\text { Similar efficacy between standard and } \\
\text { short-course RT }\end{array}$ \\
\hline \multicolumn{5}{|l|}{ Chemotherapy } \\
\hline $\begin{array}{l}\text { TMZ ( } 200 \mathrm{mg} / \mathrm{m}^{2} \text { per day for } 5 \text { days) vs. } \\
\text { standard RT ( } 60 \mathrm{~Gy} \text { in } 30 \mathrm{fr} \text { ) vs. hypofractionated } \\
\text { RT ( } 34 \mathrm{~Gy} \text { in } 10 \mathrm{fr} \text { ) }\end{array}$ & $\mathrm{R}$ & $\geq 60$ & $\geq 60$ & $\begin{array}{l}\text { Similar efficacy between hypofractionated } \\
\text { RT and TMZ }\end{array}$ \\
\hline $\begin{array}{l}\text { Dose-dense TMZ ( } 100 \mathrm{mg} / \mathrm{m}^{2} \text { per day, for days } \\
1-7 \text { and } 15-21) \text { vs. RT ( } 60 \mathrm{~Gy} \text { in } 30 \mathrm{fr})\end{array}$ & $\mathrm{R}$ & $\geq 65$ & $\geq 60$ & Noninferiority of TMZ compared with RT \\
\hline TMZ (150-200 mg/m² per day for 5 days) & $\mathrm{nR}$ & $\geq 70$ & $\leq 60$ & $\begin{array}{l}\text { Acceptable tolerance and suggested } \\
\text { benefit on functional status and survival }\end{array}$ \\
\hline $\begin{array}{l}\left.\text { TMZ (130-150 mg } / \mathrm{m}^{2} \text { per day for } 5 \text { days }\right) \text { and } \\
\text { BVZ (10 mg/kg) }\end{array}$ & $\mathrm{nR}$ & $\geq 70$ & $\leq 60$ & $\begin{array}{l}\text { Acceptable tolerance and suggested } \\
\text { benefit on functional status and survival } \\
\text { (not different from TMZ alone) }\end{array}$ \\
\hline
\end{tabular}

Abbreviations: BVZ, bevacizumab; fr, fractions; KPS, Karnofsky performance score; $\mathrm{nR}$, nonrandomized; OS, overall survival; PFS, progression-free survival; $\mathrm{R}$, randomized; $\mathrm{RT}$, radiotherapy; $\mathrm{TMZ}$, temozolomide.

(5.1 months in the group of patients receiving the standard schedule versus 5.6 months in the group of patients treated with the shorter course). Moreover, apart from the reduced treatment time, patients receiving the abbreviated regimen had a lower rate of premature discontinuation of radiotherapy (10\% versus $26 \%$ ) and required fewer increments of posttreatment corticosteroid dosage ( $23 \%$ versus $49 \%)$, hence suggesting an improved tolerance. Still, this randomized study was not powered sufficiently to conclude that both treatments were equivalent. In addition to this major limitation, the biological effects of the two radiation regimens are not strictly comparable [62]. However, notwithstanding the above limitations and those from other small retrospective and single-arm prospective series evaluating this issue, hypofractionated radiotherapy appears to be safe and feasible for elderly patients with GBM [62].

\section{Chemotherapy and Targeted Therapies}

Because of concerns not only about the toxicity but also about the disadvantages associated with radiation therapy in older patients, chemotherapy with temozolomide has been studied as an alternative. Its oral administration and favorable toxicity profile might seem more convenient, especially for those patients with greater neurological and functional impairment. Some retrospective studies have suggested that upfront treatment with temozolomide might be as effective as radiotherapy in this cohort [64-66].

This issue has been recently investigated by two large randomized phase III trials [45, 67]. The three-armed Nordic Clinical Brain Tumor Study Group trial compared single-agent temozolomide $\left(200 \mathrm{mg} / \mathrm{m}^{2}\right.$ for 5 consecutive days every 28 days) with two different radiation schedules, that is, standard (60 Gy delivered in 30 fractions over 6 weeks) and hypofractionated (34 Gy administered in 10 fractions over 2 weeks) radiotherapy [67]. A total of 342 GBM patients aged 60 years or older were enrolled in the study. Median OS was significantly longer with upfront temozolomide compared with standard radiotherapy (8.3 months versus 6.0 months). No differences were found between temozolomide and hypofractionated radiotherapy (7.5 months) or between hypofractionated and standard radiotherapy. For the subgroup of patients older than 70 years, survival was significantly better with both temozolomide ( 9.0 months) and hypofractionated radiotherapy (7.0 months) than with standard radiotherapy (5.2 months). Also, treatment with temozolomide was associated with longer survival in patients whose tumors had MGMT promoter methylation compared with those without it (9.7 versus 6.8 months). MGMT promoter methylation did not have a significant effect on the response to radiotherapy.

The German Neuro-oncology Working Group noninferiority phase III trial included 373 patients aged 65 and older with AA (11\%) or GBM (89\%) [45]. The patients were randomized to receive either a dose-intensified temozolomide regimen ( $100 \mathrm{mg} / \mathrm{m}^{2}$ for 7 days every 14 days) or standard radiotherapy (60 Gy in 30 fractions over 6 weeks). Median OS times were similar in both groups $(8.6$ months for the temozolomide arm versus 9.6 months for the radiotherapy one), matching the noninferiority defined margin for temozolomide. MGMT promoter methylation, which was tested in $53 \%$ of the included patients, was associated with longer OS. Median event-free survival did not differ between both treatment groups but was longer in patients with MGMT promoter methylation who received temozolomide than in those who received radiotherapy, whereas the opposite was true for patients with the unmethylated MGMT promoter. In regards to toxicity, grade 3 or 4 hematologic adverse events were generally more frequent in the dose-dense temozolomide group.

These two studies are important large trials that reveal valuable data concerning the management of elderly patients with HGG, especially GBM. However, caution is needed in extrapolating their results because of the noninferiority design (with a large margin) and treatment crossover upon 
progression in the second study and because they both differed significantly from the standard chemoradiation regimen used for younger patients [68].

From a practical point of view, bedridden and dependent elderly patients with GBM might benefit better from chemotherapy alone [69]. Radiotherapy, even when administrated in abbreviated courses, requires daily trips to the hospital and results in increased fatigue: it is therefore considered inconvenient for these severely disabled patients with such short survival expectancy. In this setting, a phase II nonrandomized multicenter trial showed that chemotherapy with temozolomide alone in GBM patients aged 70 or older and with a KPS under $70 \%$ allowed one-quarter of these patients to become capable of self-care [70]. The encouraging median survival of 25 weeks far exceeded the 12 -week median survival assumed for a similar patient population treated with the best supportive care alone. This trial also confirmed that temozolomide had an acceptable toxicity profile even in patients with poor KPS, with grade 3 and 4 hematological toxicity comparable to that observed in younger and fitter patients. Although such results should be validated in a randomized trial, they suggest that temozolomide alone might be a useful alternative for those severely impaired patients to whom symptomatic care is still commonly offered as the sole therapeutic option.

Recently, another multicenter phase II trial evaluated the efficacy and safety of upfront temozolomide $\left(130-150 \mathrm{mg} / \mathrm{m}^{2}\right.$ for 5 days every 4 weeks) plus bevacizumab $(10 \mathrm{mg} / \mathrm{kg}$ every 2 weeks) in an elderly GBM population with poor performance status [71]. Sixty-six patients aged 70 or older with a KPS below $70 \%$ were included. Preliminarily reported median OS was 24 weeks, and $38 \%$ of the patients became transiently capable of self-care. These results are very similar to those obtained in the above-mentioned previous trial on temozolomide alone, thus confirming that temozolomide-based treatment can help these patients and suggesting that the addition of upfront bevacizumab does not appear to confer additional benefits, a result that does not suggest the need for phase III evaluation.

\section{Combined Chemoradiotherapy}

In 2005, the European Organization for Research and Treatment of Cancer (EORTC) and the National Cancer Institute of Canada ( $\mathrm{NCIC}$ ) undertook a randomized phase III trial that demonstrated that the addition of concomitant and adjuvant chemotherapy with temozolomide to postoperative radiotherapy significantly improved the survival of GBM patients as compared with radiation therapy alone (14.6 months versus 12.1 months), with these benefits lasting through 5 years of follow-up $[72,73]$. Although no differences in median OS were observed in patients aged $61-70$ years, the long-lasting benefit of combined therapy was also recorded in that subset of older patients [73]. However, because the trial included only patients aged $18-70$ years, this multimodality treatment scheme became the standard of care only for nonelderly GBM patients. As a result, the question that arises in the management of older patients with GBM is whether adding this alkylating and radiosensitizing agent during and after radiotherapy also improves survival in such patients when compared with radiation alone. However, the main disquietude about transferring this chemoradiation regimen to the elderly is the risk of neurological and hematological toxicity, which could impair the quality of life of these patients [21]. Whether adding temozolomide to radiotherapy may increase the risk of neurocognitive deficits (especially in the many patients with silent cerebrovascular disease or mild cognitive impairment) is still an unresolved question [74, 75].

In terms of survival, several retrospective studies [9, 75-80] and a meta-analysis [81] have shown promising benefits in elderly patients treated with this combined approach, with survival times ranging from 10 to 14 months. Among older patients, those with low comorbidity and more favorable prognostic factors (such as extensive resection and good performance status) appear to benefit the most $[9,82]$. Nevertheless, high rates of neurologic treatment-related toxicity, up to $40 \%-56 \%$, have been reported $[76-78,80]$.

Recently, a unique multicenter nonrandomized phase II trial specifically evaluated the efficacy and safety of an abbreviated course of radiotherapy with concomitant and adjuvant temozolomide in 71 elderly GBM patients [83]. A promising 12-month median OS was observed. The planned course of radiation therapy was completed for all the patients. Grade 3 and 4 neutropenia and thrombopenia occurred in $15 \%$ of the patients, and notable neurologic treatment-related toxicity was reported only in one patient during the adjuvant phase. Importantly, this therapeutic scheme was associated with maintenance or even improvement of most of the evaluated health-related quality of life domains and neurocognitive functions [84].

Definite results concerning the efficacy and safety of combined radiochemotherapy with temozolomide in elderly patients with GBM, as well as its impact on quality of life and cognition, need to be validated by a randomized phase III trial. Such a randomized clinical trial comparing short-course radiotherapy with or without temozolomide in GBM patients aged 65 or older, conducted by the NCIC and the EORTC, has been recently completed and will soon provide answers to the aforementioned questions.

\section{Molecular Signatures in Elderly Patients With High-Grade Gliomas}

Advanced age is accepted as one of the most pejorative prognostic factors in patients with HGG. However, the more dismal outcome attributed to older HGG patients does not appear to be explained only by more frequently associated comorbidities or by an age-related suboptimal pattern of care in such older patients [85]. In fact, there is some evidence suggesting that tumorigenic pathways to GBM vary with the age of the patient [86]. A pioneer retrospective study including $140 \mathrm{GBM}$ patients demonstrated that the prognostic effects of key genomic alterations, such as the TP53 mutation, EGFR amplification, $C D K N 2 A / p 16$ alterations, and the loss of chromosome $1 \mathrm{p}$, were dependent on the age of the patients. EGFR amplification and the loss of chromosome $1 \mathrm{p}$ had positive prognostic effects in elderly patients, whereas TP53 and $C D K N 2 A / p 16$ alterations had a negative impact on the survival rate of these patients [87]. Notably, the impact on survival of TP53 and EGFR alterations in older patients was the opposite of the observed effect in their younger counterparts; this latter finding has also been confirmed in other further recent analyses $[88,89]$. Other recent studies have shown that 
favorable prognostic biomarkers, such as the IDH mutation or G-CIMP (glioma-CpG island methylator phenotype), are virtually absent in malignant astrocytic tumors of the elderly, which may partly account, to a small extent, for the worsened prognosis of such patients [86]. Nevertheless, the fact that older patients in a large majority of cases have poorer outcomes compared with younger patients, despite similar profiles of genomic alterations, hints at the existence of thus far unknown molecular prognostic factors in the elderly [86]. In addition, other host-derived factors, such as age-related immune alterations, might play an important role in the poor prognosis observed in these patients. The term "immunosenescence" refers to an age-associated decreased immune competence that renders older individuals more prone to disease, including cancer. Although its mechanisms remain unclear, this decline of immune potential, especially adaptive immunity, might likely contribute to the progression and worse prognosis of GBM in the elderly [90].

The term "immunosenescence" refers to an ageassociated decreased immune competence that renders older individuals more prone to disease, including cancer. Although its mechanisms remain unclear, this decline of immune potential, especially adaptive immunity, might likely contribute to the progression and worse prognosis of GBM in the elderly.

In contrast to what happens with IDH1 mutations, the MGMT promoter methylation status does not seem to be agedependent $[86,91]$. The MGMT promoter methylation is at least as common in elderly GBM patients as in younger individuals and has a similar positive impact on survival in elderly patients treated with the alkylating agent temozolomide alone or in combination with radiotherapy $[45,67,68,78$, 91-94]. On the basis of previously mentioned recent prospective trials, some authors suggest that treatment of elderly patients should be tailored according to the MGMT promoter status of the tumor, favoring temozolomide in patients with the methylated promoter and radiation therapy in unmethylated patients $[45,95,96]$. Nevertheless, in the current context of a lack of alternative therapies, this proposal remains questionable so far. On the one hand, some patients with unmethylated tumors do also respond to alkylating chemotherapy [70]. On the other hand, testing the MGMT promoter methylation status is still challenging, because the techniques are not well optimized and standardized [97]. Nowadays, the key issue is in fact whether combined chemoradiation improves survival as compared with either modality alone without impairing cognition and quality of life. If radiochemotherapy is proved superior, it remains to be seen whether the benefit is exclusively restricted to MGMT promoter methylated patients or whether unmethylated patients also experience some modest benefit from this regimen, as shown in younger patients.

\section{CONCLUSION}

The optimal management of gliomas in the elderly, especially low-grade and anaplastic tumors, remains unsettled. Further specific prospective studies should address the role for surgery, the timing for radiotherapy, and the usefulness of chemotherapy in these later grade 2 and 3 gliomas. In terms of GBM, questions regarding the benefit of an extensive surgical approach and the choice of either radiation alone versus combined chemoradiotherapy at the onset of care (as well as the tolerance of such a combined approach) are yet unresolved. Ongoing clinical trials addressing these issues will provide useful answers to these topics and will thus improve the pattern of care of these patients. Additionally, the absence of age limits in future clinical trials assessing novel therapies in both young and old patients should be mandatory. However, already existing data obtained from recent trials have underlined the notion that active treatment of elderly patients might be of substantial benefit. The continuous increasing rise in the proportion of elderly people commits the medical community to developing and validating specific therapeutic regimens for these patients, with special attention to quality of life, cognitive and functional status, and social support.

\section{Author Contributions}

Conception/Design: Jaime Gállego Pérez-Larraya, Jean-Yves Delattre

Provision of study material or patients: Jaime Gállego Pérez-Larraya, Jean-Yves Delattre

Collection and/or assembly of data: Jaime Gállego Pérez-Larraya, Jean-Yves Delattre

Data analysis and interpretation: Jaime Gállego Pérez-Larraya, Jean-Yves Delattre

Manuscript writing: Jaime Gállego Pérez-Larraya, Jean-Yves Delattre

Final approval of manuscript: Jaime Gállego Pérez-Larraya, Jean-Yves Delattre

\section{Disclosures}

Jean-Yves Delattre: French National Institute of Cancer (RF). The other author indicated no financial relationships.

(C/A) Consulting/advisory relationship; (RF) Research funding; (E) Employment; (ET) Expert

testimony; (H) Honoraria received; (OI) Ownership interests; (IP) Intellectual property rights/

inventor/patent holder; (SAB) Scientific advisory board

\section{REFERENCES}

1. Delafuente JC. The silver tsunami is coming: Will pharmacy be swept away with the tide? Am J Pharm Educ 2009;73:1.

2. Bartels SJ, Naslund JA. The underside of the silver tsunami: Older adults and mental health care. N Engl J Med 2013;368:493-496.

3. Institute of Medicine. Delivering High Quality Cancer Care: Charting a New Course for a System in Crisis. Washington, D.C.: National Academies Press, 2013

4. Ostrom QT, Gittleman H, Farah P et al. CBTRUS statistical report: Primary brain and central nervous system tumors diagnosed in the United States in 2006-2010. Neuro Oncol 2013;15(suppl 2):ii1-ii56.

5. Hess KR, Broglio KR, Bondy ML. Adult glioma incidence trends in the United States, 1977-2000. Cancer 2004;101:2293-2299.

6. Hoffman S, Propp JM, McCarthy BJ. Temporal trends in incidence of primary brain tumors in the United States, 1985-1999. Neuro Oncol 2006;8:27-37.

7. Chakrabarti I, Cockburn M, Cozen W et al. A population-based description of glioblastoma multiforme in Los Angeles County, 1974-1999. Cancer 2005;104:2798-2806
8. Elia-Pasquet $S$, Provost $D$, Jaffré $A$ et al. Incidence of central nervous system tumors in Gironde, France. Neuroepidemiology 2004;23:110-117.

9. Balducci L, Colloca G, Cesari M et al. Assessment and treatment of elderly patients with cancer. Surg Oncol 2010;19:117-123.

10. Fried LP, Hall WJ. Editorial: Leading on behalf of an aging society. J Am Geriatr Soc 2008;56 1791-1795.

11. Laperriere N, Weller M, Stupp R et al. Optimal management of elderly patients with glioblastoma. Cancer Treat Rev 2013;39:350-357. 
12. Li J, Wang $M$, Won $M$ et al. Validation and simplification of the Radiation Therapy Oncology Group recursive partitioning analysis classification for glioblastoma. Int J Radiat Oncol Biol Phys 2011; 81:623-630.

13. Hutchins LF, Unger JM, Crowley JJ et al. Underrepresentation of patients 65 years of age or older in cancer-treatment trials. N Engl J Med 1999; 341:2061-2067.

14. Gross $\mathrm{CP}$, Herrin J, Wong $\mathrm{N}$ et al. Enrolling older persons in cancer trials: The effect of sociodemographic, protocol, and recruitment center characteristics. J Clin Oncol 2005;23:4755-4763.

15. Yee KW, Pater JL, Pho Let al. Enrollment of older patients in cancer treatment trials in Canada: Why is age a barrier? J Clin Oncol 2003;21:1618-1623.

16. Barnholtz-Sloan JS, Williams VL, Maldonado JL et al. Patterns of care and outcomes among elderly individuals with primary malignant astrocytoma. $J$ Neurosurg 2008;108:642-648.

17. Scott J, Tsai YY, Chinnaiyan P et al. Effectiveness of radiotherapy for elderly patients with glioblastoma. Int J Radiat Oncol Biol Phys 2011;81:206-210.

18. Kita D, Ciernik IF, Vaccarella $S$ et al. Age as a predictive factor in glioblastomas: Populationbased study. Neuroepidemiology 2009;33:17-22.

19. Gulati $S$, Jakola AS, Johannesen TB et al. Survival and treatment patterns of glioblastoma in the elderly: A population-based study. World Neurosurg 2012;78:518-526.

20. Aapro MS, Köhne $\mathrm{CH}$, Cohen $\mathrm{HJ}$ et al. Never too old? Age should not be a barrier to enrollment in cancer clinical trials. The Oncologist 2005;10: 198-204.

21. Gállego Pérez-Larraya J, Delattre JY. Treating glioblastoma in the elderly. CNS Oncol 2012;1: 193-201.

22. Pruitt AA. Medical management of patients with brain tumors. Curr Treat Options Neurol 2011 13:413-426.

23. Ricard D, Idbaih A, Ducray F et al. Primary brain tumours in adults. Lancet 2012;379:1984-1996.

24. Tatsuno I, Sugiyama T, Suzuki $S$ et al. Age dependence of early symptomatic vertebral fracture with high-dose glucocorticoid treatment for collagen vascular diseases. J Clin Endocrinol Metab 2009;94:1671-1677.

25. Chevalet P, Masseau-Imbert A, Durand-Fix MH et al. [Giant cell arteritis after the age of 75]. Ann Med Interne (Paris) 2002;153:373-377.

26. Drappatz J, Schiff D, Kesari $S$ et al. Medical management of brain tumor patients. Neurol Clin 2007;25:1035-1071, ix.

27. Vélez L, Selwa LM. Seizure disorders in the elderly. Am Fam Physician 2003;67:325-332.

28. Waterhouse E, Towne A. Seizures in the elderly: Nuances in presentation and treatment. Cleve Clin Med 2005;72(suppl 3):S26-S37.

29. Glantz MJ, Cole BF, Forsyth PA et al. Practice parameter: Anticonvulsant prophylaxis in patients with newly diagnosed brain tumors: Report of the Quality Standards Subcommittee of the American Academy of Neurology. Neurology 2000;54:18861893.

30. Arif $H$, Buchsbaum R, Pierro J et al. Comparative effectiveness of 10 antiepileptic drugs in older adults with epilepsy. Arch Neurol 2010;67:408-415.

31. Zeber JE, Copeland LA, Pugh MJ. Variation in antiepileptic drug adherence among older patients with new-onset epilepsy. Ann Pharmacother 2010; 44:1896-1904.

32. Contin M, Mohamed S, Albani F et al. Levetiracetam clinical pharmacokinetics in elderly and very elderly patients with epilepsy. Epilepsy Res 2012;98: 130-134.

33. Gehring K, Patwardhan SY, Collins R et al. A randomized trial on the efficacy of methylphenidate and modafinil for improving cognitive functioning and symptoms in patients with a primary brain tumor. J Neurooncol 2012;107:165-174.

34. Minton $O$, Richardson $A$, Sharpe $M$ et al Psychostimulants for the management of cancerrelated fatigue: A systematic review and metaanalysis. J Pain Symptom Manage 2011;41:761767.

35. Yennurajalingam S, Palmer JL, Chacko R et al Factors associated with response to methylphenidate in advanced cancer patients. The Oncologist 2011;16:246-253.

36. Berger $A$. Treating fatigue in cancer patients. The Oncologist 2003;8(suppl 1):10-14.

37. Kaloshi G, Psimaras D, Mokhtari $K$ et al. Supratentorial low-grade gliomas in older patients. Neurology 2009;73:2093-2098.

38. Wrensch M, Rice T, Miike R et al. Diagnostic, treatment, and demographic factors influencing survival in a population-based study of adult glioma patients in the San Francisco Bay Area. Neuro Oncol 2006;8:12-26.

39. Iwamoto FM, Reiner AS, Nayak L et al. Prognosis and patterns of care in elderly patients with glioma. Cancer 2009;115:5534-5540.

40. Pouratian N, Mut M, Jagannathan J et al. Lowgrade gliomas in older patients: A retrospective analysis of prognostic factors. J Neurooncol 2008 90:341-350.

41. Schomas DA, Laack NN, Brown PD. Low-grade gliomas in older patients: Long-term follow-up from Mayo Clinic. Cancer 2009;115:3969-3978.

42. Pignatti $F$, van den Bent $M$, Curran $D$ et al Prognostic factors for survival in adult patients with cerebral low-grade glioma. J Clin Oncol 2002;20: 2076-2084.

43. Karim AB, Maat $B$, Hatlevoll $R$ et al. $A$ randomized trial on dose-response in radiation therapy of low-grade cerebral glioma: European Organization for Research and Treatment of Cancer (EORTC) Study 22844. Int J Radiat Oncol Biol Phys 1996;36:549-556.

44. Keime-Guibert $F$, Chinot $O$, Taillandier $L$ et al. Radiotherapy for glioblastoma in the elderly. N EngI J Med 2007:356:1527-1535.

45. Wick W, Platten M, Meisner C et al. Temozolomide chemotherapy alone versus radiotherapy alone for malignant astrocytoma in the elderly: The NOA-08 randomised, phase 3 trial. Lancet Oncol 2012;13:707-715.

46. Tanaka S, Meyer FB, Buckner JC et al. Presentation, management, and outcome of elderly patients with newly-diagnosed anaplastic astrocytoma. J Neurooncol 2012;110:227-235.

47. van den Bent MJ, Brandes AA, Taphoorn M et al. Adjuvant procarbazine, lomustine, and vincristine chemotherapy in newly diagnosed anaplastic oligodendroglioma: Long-term follow-up of EORTC brain tumor group study 26951. J Clin Oncol 2013:31:344-350.

48. Ducray F, del Rio MS, Carpentier C et al. Upfront temozolomide in elderly patients with anaplastic oligodendroglioma and oligoastrocytoma. J Neurooncol 2011;101:457-462.

49. Lowry JK, Snyder JJ, Lowry PW. Brain tumors in the elderly: Recent trends in a Minnesota cohort study. Arch Neurol 1998;55:922-928.

50. Laigle-Donadey F, Sanson M. [Pattern of care of high-grade gliomas]. Rev Prat 2006;56:17791786.

51. Marijnen CA, van den Berg SM, van Duinen SG et al. Radiotherapy is effective in patients with glioblastoma multiforme with a limited prognosis and in patients above 70 years of age: A retrospective single institution analysis. Radiother Oncol 2005;75:210-216

52. Chaichana KL, Garzon-Muvdi T, Parker S et al. Supratentorial glioblastoma multiforme: The role of surgical resection versus biopsy among older patients. Ann Surg Oncol 2011;18:239-245.

53. Oszvald A, Güresir E, Setzer M et al. Glioblastoma therapy in the elderly and the importance of the extent of resection regardless of age. J Neurosurg 2012;116:357-364.

54. Ewelt C, Goeppert M, Rapp M et al. Glioblastoma multiforme of the elderly: The prognostic effect of resection on survival. J Neurooncol 2011; 103:611-618

55. Kelly PJ, Hunt C. The limited value of cytoreductive surgery in elderly patients with malignant gliomas. Neurosurgery 1994;34:62-66; discussion 66-67.

56. Pierga JY, Hoang-Xuan $K$, Feuvret $L$ et al. Treatment of malignant gliomas in the elderly. Neurooncol 1999;43:187-193.

57. Chaichana KL, Chaichana KK, Olivi A et al. Surgical outcomes for older patients with glioblastoma multiforme: Preoperative factors associated with decreased survival. Clinical article. J Neurosurg 2011;114:587-594.

58. Vuorinen V, Hinkka S, Färkkilä M et al. Debulking or biopsy of malignant glioma in elderly people: A randomised study. Acta Neurochir (Wien) 2003 145:5-10.

59. Iwamoto FM, Cooper AR, Reiner AS et al. Glioblastoma in the elderly: The Memorial SloanKettering Cancer Center Experience (1997-2007). Cancer 2009;115:3758-3766.

60. Idbaih A, Taillibert S, Simon JM et al. Short course of radiation therapy in elderly patients with glioblastoma multiforme. Cancer Radiother 2008; 12:788-792.

61. Hoegler DB, Davey P. A prospective study of short course radiotherapy in elderly patients with malignant glioma. J Neurooncol 1997;33:201-204.

62. Hingorani $M$, Colley WP, Dixit $S$ et al. Hypofractionated radiotherapy for glioblastoma: Strategy for poor-risk patients or hope for the future? $\mathrm{Br}$ Radiol 2012;85:e770-e781.

63. Roa W, Brasher PM, Bauman G et al. Abbreviated course of radiation therapy in older patients with glioblastoma multiforme: a prospective randomized clinical trial. J Clin Oncol 2004;22: 1583-1588.

64. Laigle-Donadey F, Figarella-Branger D, Chinot $O$ et al. Up-front temozolomide in elderly patients with glioblastoma. J Neurooncol 2010;99:89-94.

65. Chinot OL, Barrie M, Frauger E et al. Phase II study of temozolomide without radiotherapy in newly diagnosed glioblastoma multiforme in an elderly populations. Cancer 2004;100:2208-2214.

66. Glantz M, Chamberlain M, Liu Q et al. Temozolomide as an alternative to irradiation for elderly 
patients with newly diagnosed malignant gliomas. Cancer 2003;97:2262-2266

67. Malmström A, Grønberg BH, Marosi $C$ et al. Temozolomide versus standard 6-week radiotherapy versus hypofractionated radiotherapy in patients older than 60 years with glioblastoma: The Nordic randomised, phase 3 trial. Lancet Oncol 2012;13:916-926.

68. Holdhoff M, Rosner GL, Alcorn S et al. 'Elderly' patients with newly diagnosed glioblastoma deserve optimal care. J Neurooncol 2013;113:343-344.

69. Ducray F, Benouaich-Amiel A, Idbaih A et al. Complete response after one cycle of temozolomide in an elderly patient with glioblastoma and poor performance status. J Neurooncol 2008;88:185188.

70. Gallego Perez-Larraya J, Ducray F, Chinot O et al. Temozolomide in elderly patients with newly diagnosed glioblastoma and poor performance status: An ANOCEF phase II trial. J Clin Oncol 2011 29:3050-3055.

71. Reyes-Botero G, Honnorat J, Chinot $O$ et al. Temozolomide plus bevacizumab in elderly patients with newly diagnosed glioblastoma and poor performance status: An ANOCEF phase II trial. J Clin Oncol 2013;31(suppl):2020.

72. Stupp R, Mason WP, van den Bent MJ et al. Radiotherapy plus concomitant and adjuvant temozolomide for glioblastoma. N Engl J Med 2005;352: 987-996.

73. Stupp R, Hegi ME, Mason WP et al. Effects of radiotherapy with concomitant and adjuvant temozolomide versus radiotherapy alone on survival in glioblastoma in a randomised phase III study: 5-year analysis of the EORTC-NCIC trial. Lancet Oncol 2009; 10:459-466.

74. Combs SE, Wagner J, Bischof $M$ et al. Postoperative treatment of primary glioblastoma multiforme with radiation and concomitant temozolomide in elderly patients. Int J Radiat Oncol Biol Phys 2008; 70:987-992.

75. Sijben AE, McIntyre JB, Roldán GB et al. Toxicity from chemoradiotherapy in older patients with glioblastoma multiforme. J Neurooncol 2008;89: 97-103.

76. Minniti G, De Sanctis V, Muni R et al. Radiotherapy plus concomitant and adjuvant temozolomide for glioblastoma in elderly patients. J Neurooncol 2008;88:97-103.

77. Minniti G, Armosini V, Salvati M et al. Fractionated stereotactic reirradiation and concurren temozolomide in patients with recurrent glioblas toma. J Neurooncol 2011;103:683-691.

78. Brandes AA, Franceschi E, Tosoni $A$ et al. Temozolomide concomitant and adjuvant to radiotherapy in elderly patients with glioblastoma: Correlation with MGMT promoter methylation status. Cancer 2009;115:3512-3518.

79. Barker CA, Chang M, Chou JF et al. Radiotherapy and concomitant temozolomide may improve survival of elderly patients with glioblastoma. J Neurooncol 2012;109:391-397.

80. Gerstein J, Franz K, Steinbach JP et al. Postoperative radiotherapy and concomitant temozolomide for elderly patients with glioblastoma. Radiother Oncol 2010;97:382-386.

81. Yin AA, Zhang LH, Cheng JX et al. Radiotherapy plus concurrent or sequential temozolomide for glioblastoma in the elderly: A meta-analysis. PLoS One 2013;8:e74242.

82. Fiorentino A, Caivano $R$, Chiumento $C$ et al. Comorbidity assessment and adjuvant radiochemotherapy in elderly affected by glioblastoma. Med Oncol 2012;29:3467-3471.

83. Minniti G, Lanzetta G, Scaringi C et al. Phase II study of short-course radiotherapy plus concomitant and adjuvant temozolomide in elderly patients with glioblastoma. Int J Radiat Oncol Biol Phys 2012 83:93-99.

84. Minniti G, Scaringi C, Baldoni A et al. Healthrelated quality of life in elderly patients with newly diagnosed glioblastoma treated with short-course radiation therapy plus concomitant and adjuvant temozolomide. Int J Radiat Oncol Biol Phys 2013;86: 285-291.

85. Lutterbach J, Bartelt S, Momm F et al. Is older age associated with a worse prognosis due to different patterns of care? A long-term study of 1346 patients with glioblastomas or brain metastases. Cancer 2005;103:1234-1244.

86. Wiestler B, Claus R, Hartlieb SA et al. Malignant astrocytomas of elderly patients lack favorable molecular markers: An analysis of the NOA-08 study collective. Neuro Oncol 2013;15:1017-1026.
87. Batchelor TT, Betensky RA, Esposito JM et al. Age-dependent prognostic effects of genetic alterations in glioblastoma. Clin Cancer Res 2004;10: 228-233.

88. Simmons ML, Lamborn KR, Takahashi M et al. Analysis of complex relationships between age, p53, epidermal growth factor receptor, and survival in glioblastoma patients. Cancer Res 2001;61:11221128

89. Kleinschmidt-DeMasters BK, Lillehei KO, Varella-Garcia M. Glioblastomas in the older old. Arch Pathol Lab Med 2005;129:624-631.

90. Larbi A, Franceschi C, Mazzatti D et al. Aging of the immune system as a prognostic factor for human longevity. Physiology (Bethesda) 2008;23:64-74.

91. Yin AA, Zhang LH, Cheng JX et al. The predictive but not prognostic value of MGMT promoter methylation status in elderly glioblastoma patients: A meta-analysis. PLoS One 2014;9:e85102.

92. Gerstner ER, Yip S, Wang DL et al. Mgmt methylation is a prognostic biomarker in elderly patients with newly diagnosed glioblastoma. Neurology 2009;73:1509-1510.

93. Reifenberger G, Hentschel B, Felsberg J et al Predictive impact of MGMT promoter methylation in glioblastoma of the elderly. Int J Cancer 2012.

94. Minniti G, Salvati M, Arcella A et al. Correlation between 06-methylguanine-DNA methyltransferase and survival in elderly patients with glioblastoma treated with radiotherapy plus concomitant and adjuvant temozolomide. J Neurooncol 2011; 102:311-316.

95. Holdhoff M, Chamberlain MC. Controversies in the treatment of elderly patients with newly diagnosed glioblastoma. J Natl Compr Canc Netw 2013;11:1165-1172; quiz 1173.

96. Chamberlain MC. Treatment of newly diagnosed malignant glioma in the elderly people: New trials that impact therapy. Int J Clin Pract 2013;67: 1225-1227.

97. Berghoff AS, Stefanits $H$, Woehrer $A$ et al Clinical neuropathology practice guide 3-2013. Levels of evidence and clinical utility of prognostic and predictive candidate brain tumor biomarkers. Clin Neuropathol 2013;32:148-158.

\section{CME This article is available for continuing medical education credit at CME.TheOncologist.com.}

